\title{
Effect of endoscope on the peristaltic transport of a couple stress fluid with heat transfer: Application to biomedicine
}

https://doi.org/10.1515/nleng-2017-0166

Received December 11, 2017; revised May 4, 2018; accepted July 6, 2018.

\begin{abstract}
In this investigation, we have studied the problem of peristaltic flow with heat transfer through the gap between coaxial inclined tubes where the inner tube is rigid and the outer tube has sinusoidal wave travelling down its wall. The problem has been formulated in cylindrical coordinate system. The equations governing the flow have been simplified under the long wavelength and low Reynolds number assumptions. The exact solution is obtained for the temperature profile. The perturbation solutions for the velocity and pressure gradient are obtained for small couple stress parameter. Pressure difference per wavelength and frictional forces on the tube walls have been computed numerically. Results are demonstrated for various flow parameters. The better pumping results occur in vertical tube, while less pumping is seen in horizontal tube. The size of trapped bolus is small in triangular wave as compared to other waves. The present study has a wide range of applications in bio-medical engineering like the transport phenomenon in peristaltic micro pumps.
\end{abstract}

Keywords: Couple stress fluid; Biomedicine; Peristaltic flow; Heat transfer; Endoscope

\section{Introduction}

The study of non-Newtonian fluid flows has gained much attention of the researchers because of its many applications in physiology, technology and industry. Such fluids exhibit a non-linear relationship between the stress and the rate of strain. Most of the slurries, polymer solu-

\footnotetext{
*Corresponding Author: K. Ramesh, Department of Mathematics, Lovely Professional University, Jalandhar, 144411 India, E-mail: ramesh.katta1@gmail.com

M. Devakar, Department of Mathematics, Visvesvaraya National Institute of Technology, Nagpur 440010, India
}

tions, pharmaceutical formulations, cosmetics, toiletries, paints, biological fluids, food products, agricultural and dairy wastes are treated as non-Newtonian fluids. Couple stress fluid theory is one of the non-Newtonian fluid theories developed by Stokes in 1966. The couple stress fluid theory is one among the polar fluid theories which considers couple stresses in addition to the classical Cauchy stress. It is the simplest generalization of the classical theory of Newtonian fluids which allows for polar effects such as the presence of couple stresses and body couples (Devakar and Iyengar, 2008). Studies pertaining to the couple stress fluid behaviour are very useful, because such studies bear the potential to better explain the behaviour of rheological complex fluids, such as liquid crystals, colloidal fluids, liquid containing long-chain molecules as polymer suspensions, lubrication as well as human and animal blood (Ramzan et al., 2013). In view of enormous applications, many researchers have studied the flows of couple stress fluid in different geometries. Devakar and Iyengar (2010) have studied the run up flow of an incompressible couple stress fluid between parallel plates. Recently, Devakar et al. (2014a, b) have obtained the analytical solutions of couple stress fluid flows in parallel plate and cylindrical pipe geometries with slip boundary conditions. Srinivasacharya and Kaladhar (2014) have analyzed the mixed convection flow of chemically reacting couple stress fluid in a vertical channel with Soret and Dufour effects. Srivastava (1985) studied the effects of an axially symmetric mild stenosis on the flow of blood, when blood is represented by a couple stress fluid model. Khan et al. (2013) have investigated the approximate solution of the couple stress fluid equations in a semi-infinite rectangular channel with porous and uniformly expanding or contracting walls. Srinivasacharya and Srikanth (2012) have considered the oscillatory flow of an incompressible couple stress fluid through an annulus with mild constriction at the outer wall. Ramana Murthy and Srinivas (2014) have studied the first and second law analysis for the magnetohydrodynamic flow of two immiscible couple stress fluids between two parallel plates. 
Peristaltic motion has gained considerable importance because of its applications in physiology, engineering and industry. Peristalsis is now well known to the physiologists to be one of the major mechanisms for fluid transport in many biological systems. This mechanism is a natural cause of motion of fluids in the body of living creatures. In particular, peristaltic mechanism is involved in swallowing food through the esophagus, urine transport from kidney to bladder through the ureter, movement of lymphatic fluids in lymphatic vessels, bile flow from the gall bladder into the duodenum, movement of spermatozoa in the ductus efferentes of the male reproductive tract, ovum movement in the fallopian tube, blood circulation in the small blood vessels and the movement of the chyme in the gastrointestinal tract. Peristaltic pumping is also used in medical instruments such as heart-lung machine and blood pump machine etc. Keeping diverse applications of peristaltic flows in mind, a large number of theoretical and numerical works have been presented on this topic. Nadeem and Akram (2010) have discussed the peristaltic flow of a Jeffrey fluid in a rectangular duct. Nadeem et al. (2010) have investigated the heat and mass transfer analysis on peristaltic flow of a third order fluid in a diverging tube. Hayat et al. (2011) have studied the influence of compliant wall properties and heat transfer on the peristaltic flow of an incompressible viscous fluid in a curved channel. Wang et al. (2011) have discussed the magnetohydrodynamic flow of a micropolar fluid in a circular cylindrical tube. Abd-Alla and Abo-Dahab (2015) have studied the effects of magnetic field and rotation on peristaltic transport of a Jeffrey fluid in an asymmetric channel. Ramesh and Devakar $(2015,2017)$ have investigated the influence of heat and mass transfer on magnetohydrodynamic peristaltic flow of pseudoplastic fluid and couple stress fluid in a vertical asymmetric channel through porous medium. All the works that are mentioned above are carried out under the long wavelength and low Reynolds number assumptions. Ellahi et al. (2017a) have presented the homotopy perturbation solutions for the peristaltic flow of a magnetohydrodynamic non-Newtonian Carreau fluid in a uniform duct of rectangular cross section. Khan et al. (2016a) have used perturbation method to study the peristaltic flow of a psedoplastic fluid with variable viscosity in an asymmetric channel. Hina et al. (2015a) have given the analytical solutions for the peristaltic motion of an electrically conducting couple stress fluid in a channel with complaint walls. Shahzadi et al. (2017a) have studied the biomathematical analysis for the peristaltic flow of single wall carbon nanotubes under the impact of variable viscosity. Hina and Nadeem (2017) have considered the peristaltic flow of a Rabinowitsch fluid model in a non-uniform tube with combined effects of viscous dissipation and convective boundary conditions. The similar studies can be seen through the references Ellahi et al. (2016a, 2016b, 2017b); Bhatti et al. (2016a); Shahzadi and Nadeem (2017a) and Akram and Nadeem (2017), and the references therein.

The effects of an endoscope on the peristaltic flow is very important for medical diagnosis and it has many clinical applications. It is a very important tool for determining real reasons responsible for many problems in human organs in which the fluid are transported by peristaltic pumping. The endoscope is also like a catheter which is used in contemporary medical science. Few researchers have studied the peristaltic flow problems with endoscope in various situations. Mekheimer and Abd elmaboud (2008a) have discussed the influence of an endoscope on the peristaltic flow of a couple stress fluid in an annulus. Tripathi (2011) presented the effect of endoscope on peristaltic transport of fractional Maxwell fluids through the gap between two concentric uniform tubes. Nadeem et al. (2011) have studied the peristaltic flow of a Walters B fluid in an endoscope. Nagarani and Lewis (2012) have discussed the peristaltic transport of a Casson fluid through the annular gap between two coaxial tubes. Bhatti et al. (2016b) have used homotopy perturbation method to examine the effect of endoscope on the peristaltic blood flow of Sisko fluid having Titanium magnetonanoparticles through a uniform tube. Shahzadi et al. (2017b) have dealt with the combine effects of single wall carbon nanotubes and effective viscosity for the peristaltic flow of a nanofluid through annulus. Bhatti et al. (2017a) have studied the simultaneous effects of coagulation and variable magnetic field on the peristaltically induced motion of a non-Newtonian Jeffrey nanofluid containing gyrotactic microorganism through an annulus. Many investigations (Abd-Alla et al., 2015; Akbar, 2015; Hayat et al., 2017a, b) can be seen relevant to the endoscopic problems with various effects and the references therein.

Heat transfer analysis is important especially in the case of non-Newtonian physiological flows. Heat transfer involve many intricate processes like heat conduction in tissues, heat perfusion in arterial-venous blood, metabolic heat generation and external interactions such as electromagnetic radiation emitted from cell phones. It is also helpful in the treatment of diseases like removal of undesirable tissues in cancer. In view of this, many investigators have attempted heat transfer analysis in diverse situations in peristaltic transport. Vajravelu et al. (2007) have presented the peristaltic flow and heat transfer in a vertical porous annulus. Mekheimer and Abd elmaboud (2008b) have investigated the influence of heat transfer and magnetic field on peristaltic transport of a Newto- 
nian fluid in a vertical annulus. Nadeem and Akbar (2010, 2011) have studied the influence of temperature dependent viscosity on peristaltic transport of a Newtonian fluid in an endoscope and the influence of heat transfer and variable viscosity in vertical porous annulus with peristalsis. Shahzadi and Nadeem (2017b) have presented the closed form solutions for the peristaltic flow of a nanofluid in an annular region of inclined annulus. Bhatti et al. (2016c) have investigated the heat transfer analysis on clot blood model of the particle-fluid suspension through a non-uniform annulus. Khan et al. (2016b) have studied the effects of magnetohydrodynamic peristaltic flow of Walters B fluid in an inclined asymmetric channel using regular perturbation method. Hina et al. (2015b) have discussed the simultaneous effects of heat and mass transfer on the peristaltic transport of an electrically conducting couple stress fluid in a channel. Hina et al. (2016a) have used the perturbation approach to study the peristaltic flow of a Powell-Eyring fluid in a curved channel with heat transfer. Hina et al. (2016b) have explored the peristaltic flow of a Powell-Eyring fluid through curved passage with heat and mass transfer analysis. Bhatti et al. (2017b) have analysed the heat and mass transfer with the transverse magnetic field on the peristaltic motion of two-phase flow through a planar channel. Ijaz and Nadeem (2017) have addressed Cu-nanoparticles application in an inclined stenosed elastic artery with balloon model examination.

In this paper, the effect of heat transfer on the peristaltic transport of couple stress fluid in an inclined tube with endoscope is considered. It is due to many ducts in physiological systems are neither horizontal nor vertical but have some inclination with the axis. Moreover, the small glandular ducts and other tracts of the body are cylindrical in shape, so that we have considered the cylindrical geometry for the present problem. The problem has been formulated under the long wavelength and low Reynolds number assumptions and a perturbation solution is obtained for velocity and pressure gradient. The pressure difference and frictional forces at the walls have been calculated using numerical integration. The effect of flow parameters on the fluid velocity, pressure gradient, pressure difference, frictional forces and the temperature are analyzed and results are illustrated through graphs.

\section{Formulation of the problem}

Consider the peristaltic flow of an incompressible couple stress fluid in an inclined tube with an inserted endoscope. The flow is generated by sinusoidal wave train propagat- ing with constant speed $c$ along the wall of the tube. The endoscope and peristaltic tubes are treated as concentric inner and outer tubes respectively. We choose cylindrical coordinates $(R, Z)$ such that $Z$ - axis is along the center line of the tubes and $R$ is the distance measured radially (see figure 1). The temperature of the inner and outer tubes are $T_{1}$ and $T_{0}$ respectively. The walls of inner and outer tube are respectively defined as (Ramesh and Devakar, 2016)

$$
\begin{gathered}
r_{1}=a_{1}, \\
r_{2}=a_{2}+b \sin \frac{2 \pi}{\lambda}(Z-c t),
\end{gathered}
$$

where $a_{1}$ is the radius of the inner tube, $a_{2}$ is the radius of the outer tube at inlet, $b$ is the wave amplitude, $\lambda$ is the wavelength, $c$ is the wave speed and $t$ is the time.

The constitutive equations concerning the force stress tensor $\tau$ and the couple stress tensor $M$ that arises in the theory of couple stress fluids are given by (Srinivasacharya et al., 2009)

$$
\begin{aligned}
\tau= & \left(-P+\lambda_{1} \nabla \cdot \bar{q}\right) I+\mu\left[\nabla \bar{q}+(\nabla \bar{q})^{T}\right] \\
& +\frac{1}{2} I \times[\nabla \cdot M+\rho C], \\
M= & m I+2 \eta \nabla(\nabla \times \bar{q})+2 \eta^{\prime}(\nabla(\nabla \times \bar{q}))^{T} .
\end{aligned}
$$

In the above $m$ is $\frac{1}{3}$ trace of $M, \mu$ and $\lambda_{1}$ are the viscosity coefficients, $C$ is the body couple vector and $\eta, \eta^{\prime}$ are the couple stress viscosity coefficients. These material constants are constrained by the inequalities

$$
\mu \geq 0, \quad 3 \lambda_{1}+2 \mu \geq 0, \quad \eta \geq 0, \quad\left|\eta^{\prime}\right| \leq \eta .
$$

Using the equations (3) and (4), the governing equations (in the laboratory frame) for the inclined peristaltic motion of an incompressible couple stress fluid in the cylindrical polar coordinates $(R, Z)$ are

$$
\begin{gathered}
\frac{\partial U}{\partial R}+\frac{U}{R}+\frac{\partial W}{\partial Z}=0 \\
\rho\left(\frac{\partial U}{\partial t}+U \frac{\partial U}{\partial R}+W \frac{\partial U}{\partial Z}\right)=-\frac{\partial P}{\partial R}+\mu \nabla^{2} U \\
-\eta \nabla^{4} U-\rho g \beta_{T}\left(T-T_{0}\right) \cos \alpha
\end{gathered}
$$

$$
\begin{aligned}
\rho\left(\frac{\partial W}{\partial t}+U \frac{\partial W}{\partial R}+W \frac{\partial W}{\partial Z}\right)= & -\frac{\partial P}{\partial Z}+\mu \nabla^{2} W \\
& -\eta \nabla^{4} W+\rho g \beta_{T}\left(T-T_{0}\right) \sin \alpha
\end{aligned}
$$




$$
\rho c_{p}\left(\frac{\partial T}{\partial t}+U \frac{\partial T}{\partial R}+W \frac{\partial T}{\partial Z}\right)=k^{\star} \nabla^{2} T+Q_{0},
$$

where $U$ and $W$ are the velocity components in $R$ and $Z$ directions respectively, $P$ is the pressure, $\rho$ is the density, $\mu$ is the viscosity coefficient, $\eta$ is the couple stress viscosity coefficient, $g$ is the gravitation due to the acceleration, $\beta_{T}$ is the thermal expansion coefficient, $T$ is the temperature, $\alpha$ is the inclination angle, $c_{p}$ is the specific heat, $k^{\star}$ is the thermal conductivity and $Q_{0}$ is the heat generation parameter.

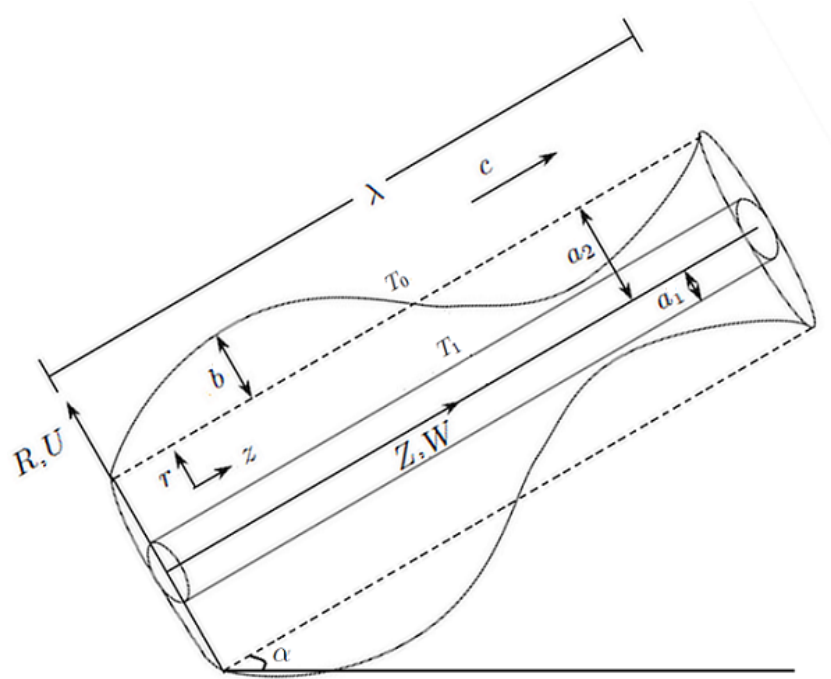

Fig. 1: Geometry of the problem.

The flow is inherently unsteady in the laboratory frame $(R, Z)$. However, the flow become steady in the wave frame $(r, z)$ moving away from the laboratory frame with speed $c$ in the direction of propagation of the wave. Taking $u$ and $w$ as the velocity components in $r$ and $z$-directions, the transformations from the laboratory frame to the wave frame are given by

$$
r=R, \quad z=Z-c t, \quad u=U, \quad w=W-c .
$$

Using the transformations (10), the governing equations (6)-(9) become

$$
\frac{\partial u}{\partial r}+\frac{u}{r}+\frac{\partial w}{\partial z}=0
$$

$\rho\left(u \frac{\partial u}{\partial r}+w \frac{\partial u}{\partial z}\right)=-\frac{\partial p}{\partial r}+\mu \nabla^{2} u-\eta \nabla^{4} u+\rho g \beta_{T}\left(\bar{T}-\bar{T}_{0}\right) \cos \alpha$

$\rho\left(u \frac{\partial w}{\partial r}+w \frac{\partial w}{\partial z}\right)=-\frac{\partial p}{\partial z}+\mu \nabla^{2} w-\eta \nabla^{4} w+\rho g \beta_{T}\left(\bar{T}-\bar{T}_{0}\right) \sin \alpha, y^{2}\left(\frac{\partial^{2}}{\partial r^{2}}+\frac{1}{r} \frac{\partial}{\partial r}\right)^{2} w-\left(\frac{\partial^{2}}{\partial r^{2}}+\frac{1}{r} \frac{\partial}{\partial r}\right) w=-\frac{\partial p}{\partial z}+G r \theta \sin \alpha$,

$$
\rho c_{p}\left(u \frac{\partial \bar{T}}{\partial r}+w \frac{\partial \bar{T}}{\partial z}\right)=k^{\star} \nabla^{2} \bar{T}+Q_{0} .
$$

Using the non-dimensional quantities (Ramesh and Devakar, 2016),

$$
\begin{gathered}
\bar{r}=\frac{r}{a_{2}}, \quad \bar{r}_{1}=\frac{r_{1}}{a_{2}}=\epsilon<1, \quad \bar{z}=\frac{z}{\lambda}, \quad \bar{u}=\frac{\lambda}{a_{2} c} u, \\
\bar{r}_{2}=\frac{r_{2}}{a_{2}}=1+\phi \sin (2 \pi z), \quad \bar{w}=\frac{w}{c}, \quad \delta=\frac{a_{2}}{\lambda}, \\
R e=\frac{\rho c a_{2}}{\mu}, \quad \bar{p}=\frac{a_{2}^{2}}{\lambda \mu c} p, \quad \bar{t}=\frac{c t}{\lambda}, \quad \phi=\frac{b}{a_{2}}<1, \\
\operatorname{Pr}=\frac{\mu c_{p}}{k^{\star}}, \quad y=\sqrt{\frac{\eta}{\mu a_{2}^{2}}}, \quad G r=\frac{\rho g a_{2}^{2} \beta_{T}\left(\bar{T}_{1}-\bar{T}_{0}\right)}{\mu c}, \\
\theta=\frac{\bar{T}_{-}-\bar{T}_{0}}{\bar{T}_{1}-\bar{T}_{0}}, \quad \beta=\frac{Q_{0} a_{2}^{2}}{k^{\star}\left(\bar{T}_{1}-\bar{T}_{0}\right)},
\end{gathered}
$$

the governing equations (11)-(14) reduced to,

$$
\begin{aligned}
\frac{\partial u}{\partial r} & +\frac{u}{r}+\frac{\partial w}{\partial z}=0 \\
\operatorname{Re} \delta^{3}\left(u \frac{\partial u}{\partial r}+w \frac{\partial u}{\partial z}\right)= & -\frac{\partial p}{\partial r}+\delta^{2}\left(\frac{\partial^{2}}{\partial r^{2}}+\frac{1}{r} \frac{\partial}{\partial r}+\delta^{2} \frac{\partial^{2}}{\partial z^{2}}\right) u \\
& -y^{2} \delta^{2}\left(\frac{\partial^{2}}{\partial r^{2}}+\frac{1}{r} \frac{\partial}{\partial r}\right. \\
& \left.+\delta^{2} \frac{\partial^{2}}{\partial z^{2}}\right)^{2} u+\delta G r \theta \cos \alpha
\end{aligned}
$$

$$
\begin{aligned}
\operatorname{Re} \delta\left(u \frac{\partial w}{\partial r}+w \frac{\partial w}{\partial z}\right)= & -\frac{\partial p}{\partial z}+\left(\frac{\partial^{2}}{\partial r^{2}}+\frac{1}{r} \frac{\partial}{\partial r}+\delta^{2} \frac{\partial^{2}}{\partial z^{2}}\right) w \\
& -y^{2}\left(\frac{\partial^{2}}{\partial r^{2}}+\frac{1}{r} \frac{\partial}{\partial r}+\delta^{2} \frac{\partial^{2}}{\partial z^{2}}\right)^{2} w \\
& +G r \theta \sin \alpha
\end{aligned}
$$

$$
\operatorname{RePr} \delta\left(u \frac{\partial \theta}{\partial r}+w \frac{\partial \theta}{\partial z}\right)=\left(\frac{\partial^{2} \theta}{\partial r^{2}}+\frac{1}{r} \frac{\partial \theta}{\partial r}+\delta^{2} \frac{\partial^{2} \theta}{\partial z^{2}}\right)+\beta
$$

The above partial differential equations are highly nonlinear and hence it is difficult to solve them analytically. Under the assumption of long wavelength and low Reynolds number the non-linear governing equations (16)-(18) reduced to,

$$
\frac{\partial p}{\partial r}=0
$$




$$
\frac{\partial^{2} \theta}{\partial r^{2}}+\frac{1}{r} \frac{\partial \theta}{\partial r}+\beta=0
$$

The non-dimensional boundary conditions to be satisfied are

$$
\begin{aligned}
& w=-1, \quad \frac{\partial^{2} w}{\partial r^{2}}=0, \quad \theta=1 \quad \text { at } \quad r=r_{1}, \\
& w=-1, \quad \frac{\partial^{2} w}{\partial r^{2}}=0, \quad \theta=0 \quad \text { at } \quad r=r_{2} .
\end{aligned}
$$

\section{Solution of the problem}

Solving equation (21) with the corresponding boundary conditions (22) and (23), the temperature profile is obtained as:

$$
\theta=-\frac{\beta}{4} r^{2}+A_{1} \ln r+A_{2},
$$

in which

$$
A_{1}=\frac{4+\beta\left(r_{1}^{2}-r_{2}^{2}\right)}{4 \ln \left(\frac{r_{1}}{r_{2}}\right)}, \quad A_{2}=1+\frac{\beta}{4} r_{1}^{2}-A_{1} \ln r_{1} .
$$

Due to the complexity of this model, to get the solution of velocity profile we have used regular perturbation method $y^{2}$ as a perturbation parameter. For perturbation solution, we express $w$ as

$$
w=w_{0}+y^{2} w_{1}+O\left(y^{4}\right) .
$$

Substituting equations (24) and (25) in equations (20), (22) and (23), and comparing the like power of $y^{2}$, we get the zeroth order and first order systems. Solving these systems with the corresponding boundary conditions, the expression for the velocity is given by

$$
\begin{aligned}
w= & \left(\frac{r^{2}}{4}+A_{4} \ln r+A_{6}\right) \frac{d p}{d z}+B_{1} r^{4}+B_{2} r^{2}+B_{3} r^{2} \ln r \\
& +A_{3} \ln r+A_{5}+y^{2}\left(B_{1} r^{4}+B_{4} r^{2}+B_{3} r^{2} \ln r\right. \\
& \left.+A_{9} \ln r+A_{10}\right) .
\end{aligned}
$$

The expression for non-dimensional volume flow rate is given by

$$
F=\int_{r_{1}}^{r_{2}} r w d r .
$$

Using equation (26) in (27), the pressure gradient can be obtained as

$$
\frac{d p}{d z}=\left(\frac{F-A_{8}}{A_{7}}\right)-y^{2}\left(\frac{A_{11}}{A_{7}}\right),
$$

where

$$
\begin{aligned}
A_{3}= & \left(B_{1}\left(r_{1}^{4}-r_{2}^{4}\right)+B_{2}\left(r_{1}^{2}-r_{2}^{2}\right)\right. \\
& \left.+B_{3}\left(r_{1}^{2} \ln r_{1}-r_{2}^{2} \ln r_{2}\right)\right) / \ln \left(r_{2} / r_{1}\right), \\
A_{4}= & \left(r_{1}^{2}-r_{2}^{2}\right) / 4 \ln \left(r_{2} / r_{1}\right), \\
A_{5}= & -1-B_{1} r_{1}^{4}-B_{2} r_{1}^{2}-B_{3} r_{1}^{2} \ln r_{1}-A_{3} \ln r_{1}, \\
A_{6}= & -A_{4} \ln r_{1}-r_{1}^{2} / 4, \\
A_{7}= & \left(r_{2}^{4}-r_{1}^{4}\right) / 16+\left(2 A_{6}-A_{4}\right)\left(r_{2}^{2}-r_{1}^{2}\right) / 4 \\
& +A_{4}\left(r_{2}^{2} \ln r_{2}-r_{1}^{2} \ln r_{1}\right) / 2, \\
A_{8}= & B_{1}\left(r_{2}^{6}-r_{1}^{6}\right) / 6+\left(4 B_{2}-B_{3}\right)\left(r_{2}^{4}-r_{1}^{4}\right) / 16 \\
& +\left(2 A_{5}-A_{3}\right)\left(r_{2}^{2}-r_{1}^{2}\right) / 4+B_{3}\left(r_{2}^{4} \ln r_{2}-r_{1}^{4} \ln r_{1}\right) / 4 \\
& +A_{3}\left(r_{2}^{2} \ln r_{2}-r_{1}^{2} \ln r_{1}\right) / 2, \\
A_{9}= & \left(B_{1}\left(r_{1}^{4}-r_{2}^{4}\right)+B_{4}\left(r_{1}^{2}-r_{2}^{2}\right)\right. \\
& \left.+B_{3}\left(r_{1}^{2} \ln r_{1}-r_{2}^{2} \ln r_{2}\right)\right) / \ln \left(r_{2} / r_{1}\right), \\
A_{10}= & -B_{1} r_{1}^{4}-B_{6} r_{1}^{2}-B_{3} r_{1}^{2} \ln r_{1}-A_{9} \ln r_{1}, \\
A_{11}= & B_{1}\left(r_{2}^{6}-r_{1}^{6}\right) / 6+\left(4 B_{4}-B_{3}\right)\left(r_{2}^{4}-r_{1}^{4}\right) / 16 \\
& +\left(2 A_{10}-A_{9}\right)\left(r_{2}^{2}-r_{1}^{2}\right) / 4+B_{3}\left(r_{2}^{4} \ln r_{2}-r_{1}^{4} \ln r_{1}\right) / 4 \\
& +A_{9}\left(r_{2}^{2} \ln r_{2}-r_{1}^{2} \ln r_{1}\right) / 2, \\
B_{1}= & G r \beta \sin \alpha / 64, \quad B_{2}=G r\left(A_{1}-A_{2}\right) \sin \alpha / 4, \\
B_{3}= & -G r A_{1} \sin \alpha / 4, \quad B_{4}=G r\left(A_{1}-A_{2}\right) \sin \alpha / 4+16 B_{1} .
\end{aligned}
$$

The pressure difference $\Delta p_{\lambda}$ and friction force $F_{\lambda}$ on inner and outer tubes $F_{\lambda}^{(i)}$ and $F_{\lambda}^{(o)}$ are given by

$$
\begin{aligned}
& \Delta p_{\lambda}=\int_{0}^{1} \frac{d p}{d z} d z ; \quad F_{\lambda}^{(i)}=\int_{0}^{1} r_{1}^{2}\left(-\frac{d p}{d z}\right) d z ; \\
& F_{\lambda}^{(o)}=\int_{0}^{1} r_{2}^{2}\left(-\frac{d p}{d z}\right) d z,
\end{aligned}
$$

where $\frac{d p}{d z}$ is given by equation (28).

\section{Results and discussion}

The focus of this section is to examine the role of various flow parameters on velocity, pressure gradient, temperature, pressure difference, frictional forces at the walls and trapping phenomenon. Fig. 2 displays the behaviour of temperature for different values of heat generation parameter $\beta$. It is depicted from the figure that, the temperature increases with increase of heat generation parameter $\beta$, and the maximum value of the temperature occurs near the middle of the peristaltic tube. It is due to fact that as heat generates during blood flow in physiological systems, there is a significant rise in thickness of the boundary layer. Thereby the temperature of the boundary layer 
enhanced by appreciable extend. The similar observation is seen in the references Ramesh and Devakar $(2015,2016)$. Fig. 3 is plotted to see the variation of velocity profile for different values of inclination angle $\alpha$, Grashof number $G r$, wave amplitude $\phi$ and volume flow rate $\Theta$. It is observed from Figs. 3(a-b) that, the velocity increases near the endoscope and decreases near the tube walls with increase in inclination angle $\alpha$ and Grashof number Gr. The buoyancy forces play a dominant role near the endoscopic tube that's why velocity profile increases near the endoscopic tube whereas reflux case or viscous forces play a dominant role near the outer wall so velocity profile contributes to decrease. The similar observation is noted in Nadeem and Hina (2015). It is depicted from the Fig. 3(c) that, the velocity decreases with increase of wave amplitude $\phi$ and the situation is reversed with increase of volume flow rate $\Theta$ (see Fig. 3(d)).

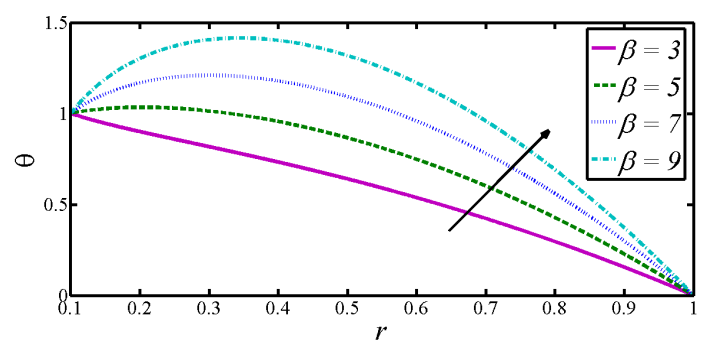

Fig. 2: The temperature profile for different values of heat generation parameter.

The pressure gradient is illustrated in Fig. 4 for different values of couple stress parameter $y$, inclination angle $\alpha$, Grashof number $G r$ and volume flow rate $\Theta$. It is noticed from these figures that, in the wider part of the tube $z \in(0,0.5)$ and $z \in(1,1.5)$, the pressure gradient is relatively small. i.e., the flow can easily pass without the imposition of a large pressure gradient. On the other hand, in the narrow part of the tube $z \in(0.5,1)$, the pressure gradient is large for given volume flow rate, that means a much larger pressure gradient is required to maintain the same flux to pass it. Moreover, it is seen from the Fig. 4(a) that, pressure gradient decreases with increase of couple stress parameter $y$. As $y=\sqrt{\eta / \mu a^{2}}$, decrease of couple stress parameter $y$ gives couple stress to Newtonian fluid model. Hence, it can be concluded that less pressure gradient is required for the flow of fluid in Newtonian fluid model than the couple stress fluid model. Fig. 4(b) depicts that, the pressure gradient is an increasing function of inclination angle $\alpha$. This shows that pressure gradient increases from horizontal to vertical tube. It is noted from Figs. 4(c- d) that, increasing of Grashof number $\mathrm{Gr}$ and volume flow rate $\Theta$ increases the pressure gradient. The similar trend is observed in Ramesh and Devakar (2016). The effects of various parameters on the pressure difference $\Delta p_{\lambda}$ are shown in Fig. 5. We split the whole graph into four regions as follows: peristaltic pumping region $\left(\Delta p_{\lambda}>0, \Theta>0\right)$, augmented pumping region $\left(\Delta p_{\lambda}<0, \Theta>0\right)$, retrograde pumping region $\left(\Delta p_{\lambda}>0, \Theta<0\right)$ and free pumping region $\left(\Delta p_{\lambda}=0\right)$. In the Peristaltic pumping region, the positive value of $\Theta$ is entirely due to the peristalsis after overcoming the pressure difference. In augmented pumping region, a negative pressure difference assists the flow due to the peristalsis of the walls. In retrograde pumping region, the flow is opposite to the direction of the peristaltic motion and in the free pumping region, the flow is caused purely by the peristalsis of the walls. It is depicted from the Fig. 5(a) that, the pumping rate increases in the retrograde pumping region and decreases in the augmented pumping region with increasing of radius ratio $\epsilon$. Pumping rate increases with increasing of inclination angle $\alpha$ and heat generation parameter $\beta$ in all the pumping regions (see Figs. 5(b-c)). It is also seen that, best pumping occurs from horizontal tube to vertical tube. It is noted from the Fig. 5(d) that, the pumping rate is a decreasing function of couple stress parameter $y$ and the best pumping can be seen in Newtonian fluid as compared to the couple stress fluid. It can be observed that, the pressure difference and volume flow rate are inversely proportional to each other. Fig. 6 describes the variations of frictional forces on the peristaltic tube. It is observed from these figures that, the frictional forces have quite opposite features when compared with the pressure difference.

Trapping is an interesting phenomenon in peristaltic motion. It is basically the formation of an internally circulating bolus of fluid by the closed streamlines. The trapped bolus pushes ahead along with the peristaltic wave. Fig. 7 shows the trapping phenomenon for different values of radius ratio $\epsilon$. It is seen that with the increase of radius ratio $\epsilon$, the size of the trapped bolus increases. Fig. 8 is plotted to see the trapping phenomenon in different peristaltic wave shapes (sinusoidal, triangular, square and trapezoidal). It is noticed that, the size of the trapped bolus in triangular wave is smaller than those in sinusoidal, square and trapezoidal waves.

\section{Conclusions}

The perturbation solution of the peristaltic transport of couple stress fluid in an inclined tube with the endoscope 


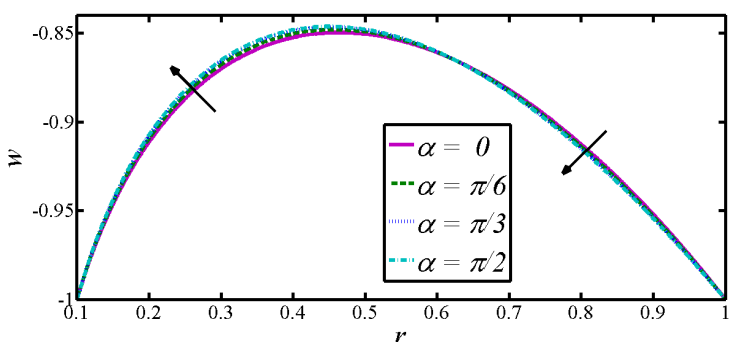

(a)

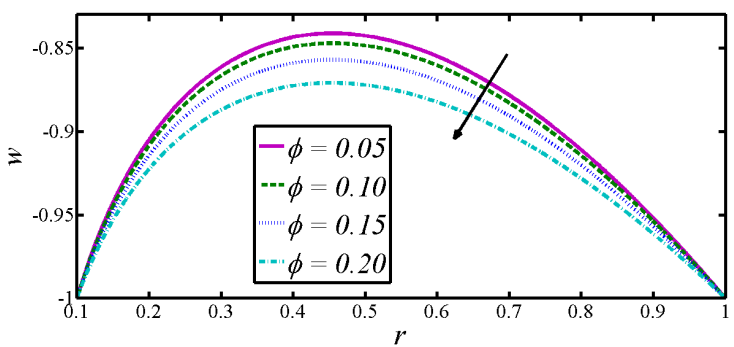

(c)

Fig. 3: The axial velocity profile for various fluid parameters.

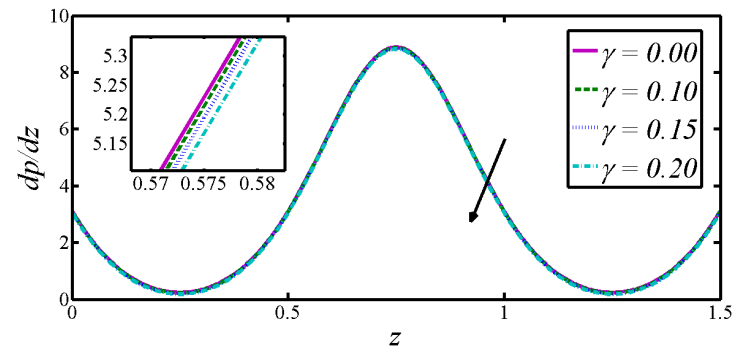

(a)

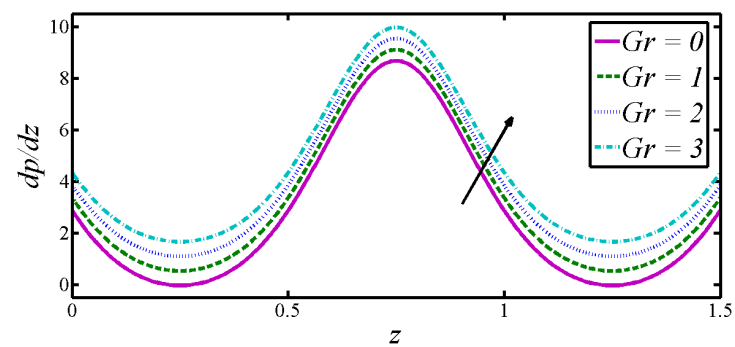

(c)

Fig. 4: The pressure gradient verses $z$ for various fluid parameters.

is obtained. The effect of heat transfer and couple stresses on the flow variables is analyzed. The pressure difference and frictional forces have been calculated numerically. The summary of the performed study is as follows:

- The temperature is an increasing function of heat generation parameter.

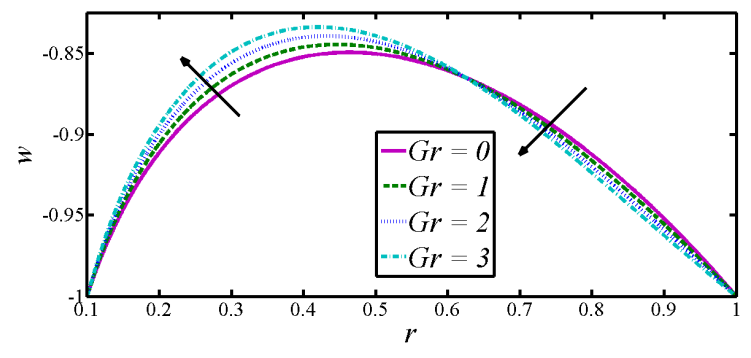

(b)

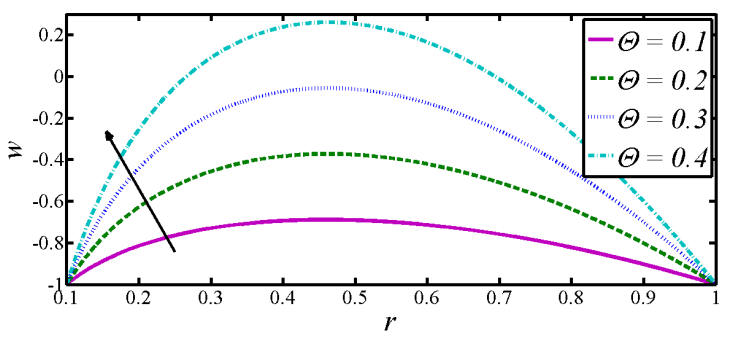

(d)

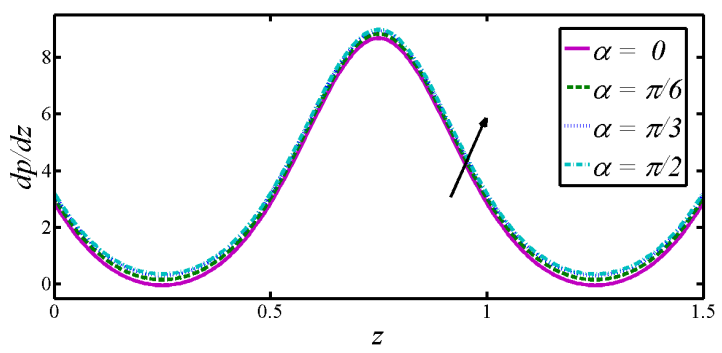

(b)

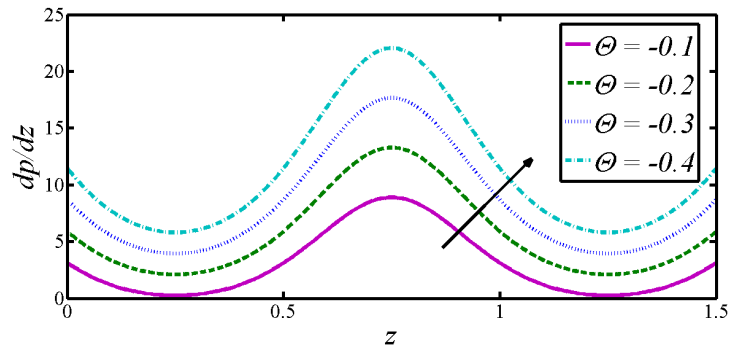

(d)

- The velocity increases near the endoscope and decreases near the peristaltic tube from horizontal tube to vertical tube.

- $\quad$ Pressure gradient decreases with increase of inclination angle, Grashof number and volume flow rate, the trend is reversed in couple stress parameter. 


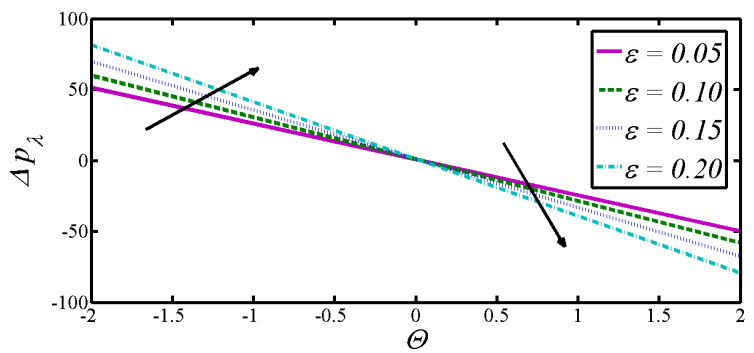

(a)

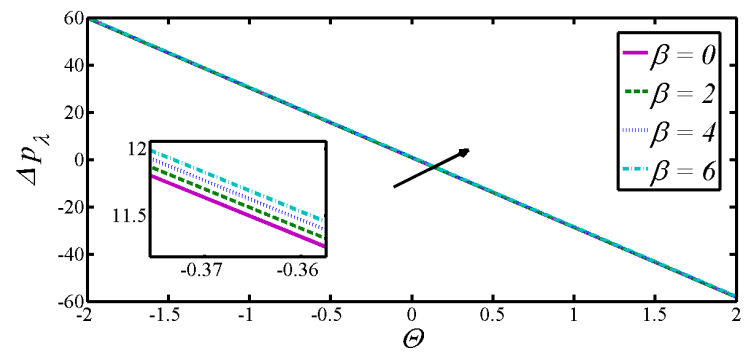

(c)

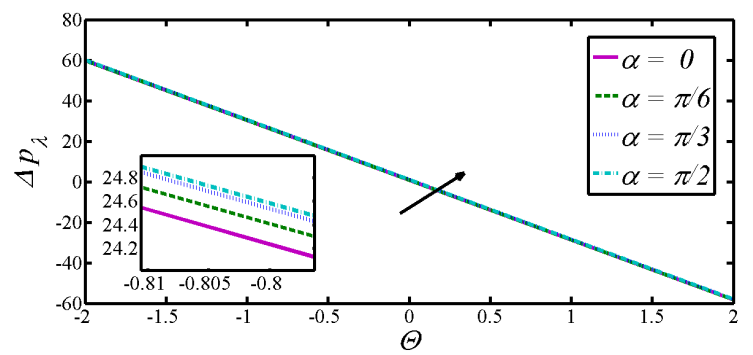

(b)

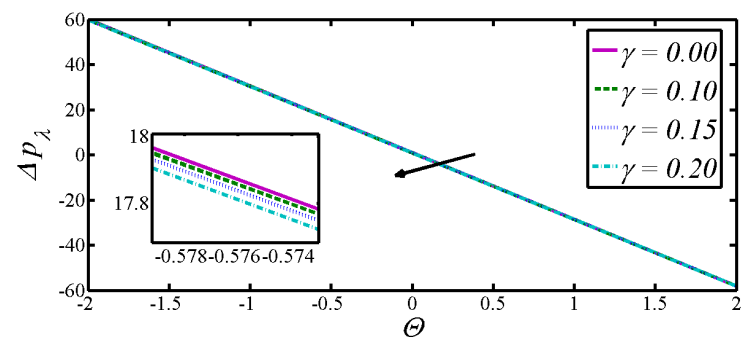

(d)

Fig. 5: The pressure difference verses volume flow rate for various fluid parameters.

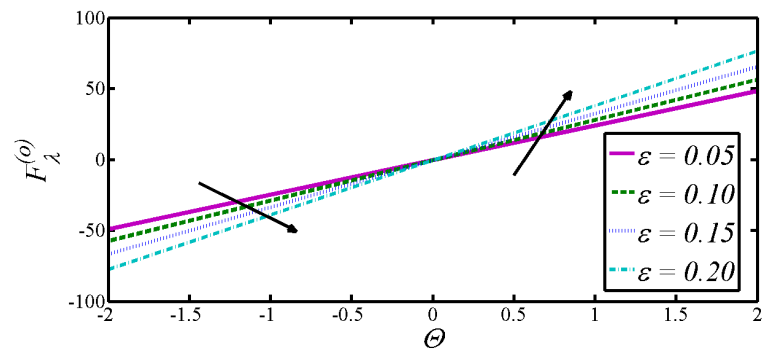

(a)

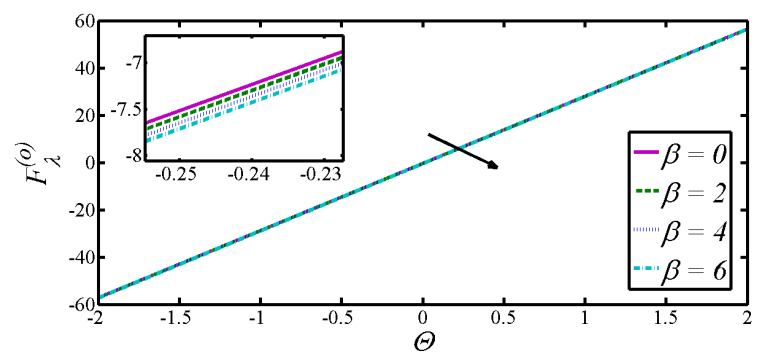

(c)

Fig. 6: The frictional forces on the outer tube verses volume flow rate for various fluid parameters.

- $\quad$ Better pumping is seen in the vertical tube as compared to the horizontal tube.

- $\quad$ The frictional force and pressure difference have quite opposite behaviour with involved parameters.

- The size of the trapped bolus increases with the increase of radius ratio and the size of trapped bolus

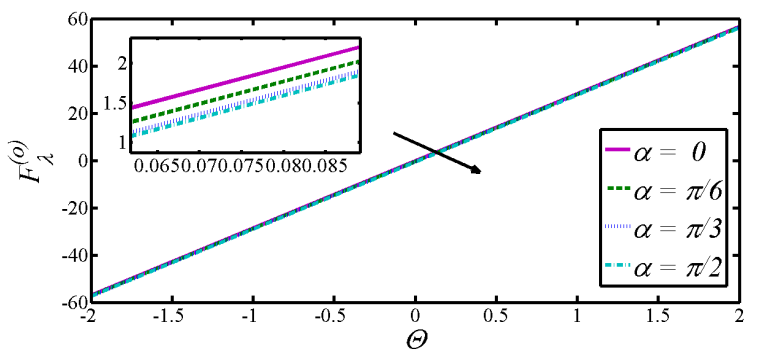

(b)

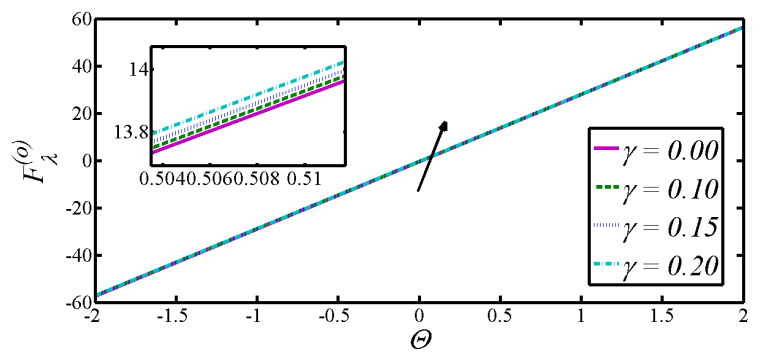

(d) 


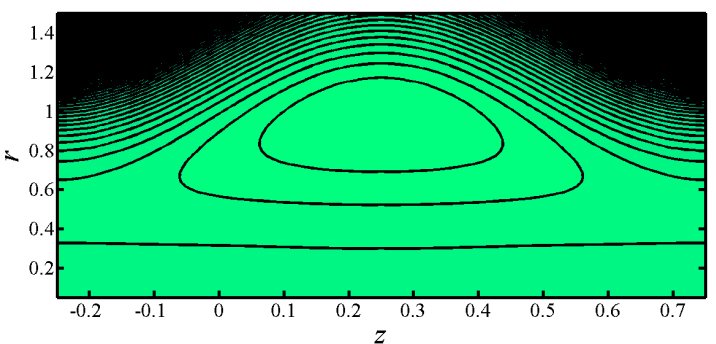

(a)

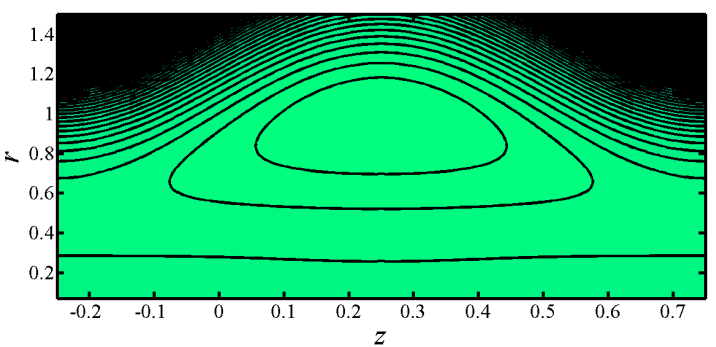

(c)

Fig. 7: Streamlines for different values of radius ratio.

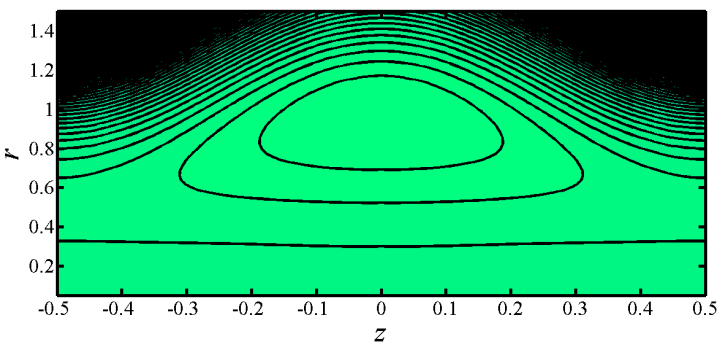

(a)

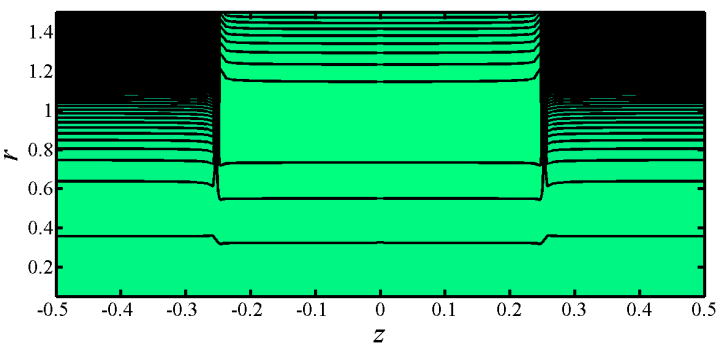

(c)

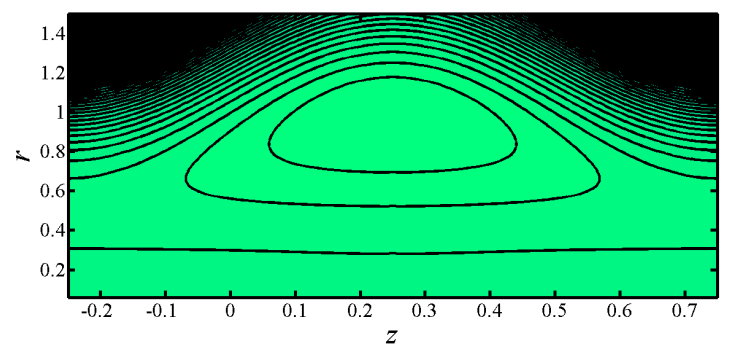

(b)

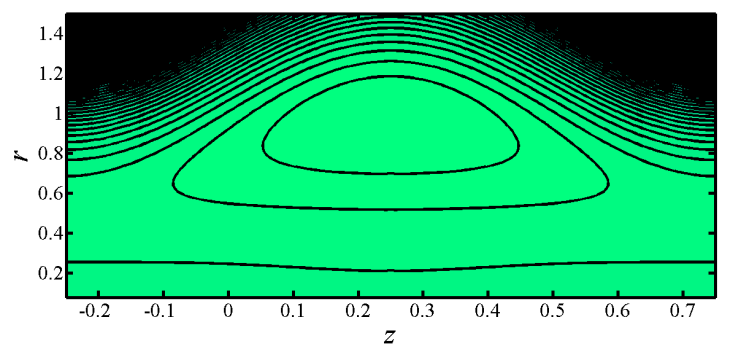

(d)

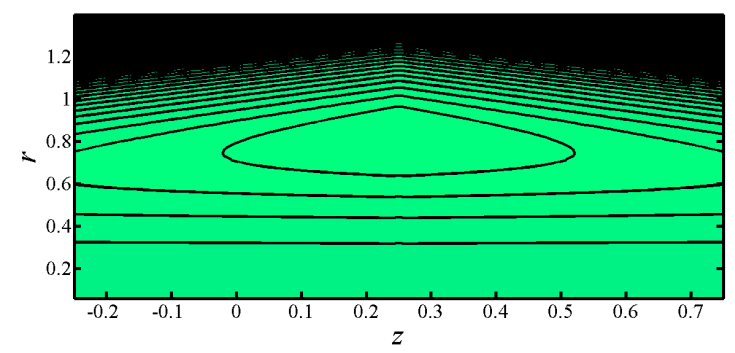

(b)

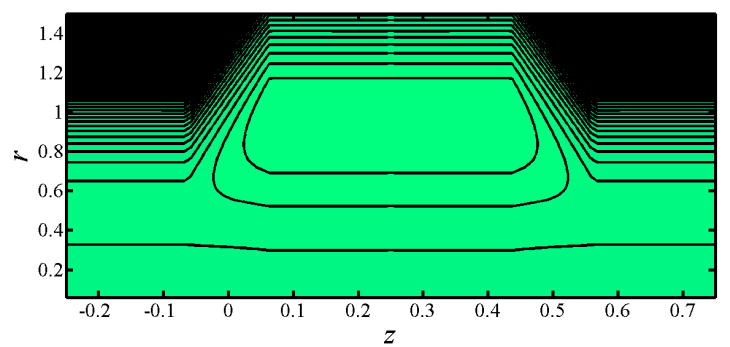

(d)

Fig. 8: Streamlines for different wave shapes such as, (a) Sinusoidal wave, (b) Triangular wave, (c) Square wave, (d) Trapezoidal wave.

Acknowledgement: The authors would like to thank the anonymous reviewers for their valuable comments and suggestions to improve the quality of the paper.

\section{References}

[1] Abd-Alla, A.M. and Abo-Dahab, S.M., "Magnetic field and rotation effects on peristaltic transport of a Jeffrey fluid in an asymmetric channel", Journal of Magnetism and Magnetic Materials. 2015, 374(15), 680-689.

[2] Abd-Alla, A.M., Abo-Dahab, S.M. and Kilicman, A., "Peristaltic flow of a Jeffrey fluid under the effect of radially varying mag- 
netic field in a tube with an endoscope", Journal of Magnetism and Magnetic Materials. 2015, 384, 79-86.

[3] Akbar, N.S. (2015), “Endoscopy analysis for the peristaltic flow of nanofluids containing carbon nanotubes with heat transfer", Zeitschrift für Naturforschung A, Vol. 70 No. 9, pp. 745-755.

[4] Akram, S. and Nadeem, S., "Influence of nanoparticles phenomena on the peristaltic flow of pseudoplastic fluid in an inclined asymmetric channel with different wave forms", Iranian Journal of Chemistry and Chemical Engineering. 2017, 36(2), 107-124.

[5] Bhatti, M.M., Ellahi, R. and Zeeshan, A., "Study of variable magnetic field on the peristaltic flow of Jeffrey fluid in a nonuniform rectangular duct having compliant walls", Journal of Molecular Liquids. 2016, 222, 101-108.

[6] Bhatti, M.M., Zeeshan, A. and Ellahi, R., "Endoscope analysis on peristaltic blood flow of Sisko fluid with Titanium magnetonanoparticles", Computers in Biology and Medicine. 2016, 78, 29-41.

[7] Bhatti, M.M., Zeeshan, A. and Ellahi, R., "Heat transfer analysis on peristaltically induced motion of particlefluid suspension with variable viscosity: Clot blood model”, Computer Methods and Programs in Biomedicine. 2016, 137, 115-124.

[8] Bhatti, M.M., Zeeshan, A. and Ellahi, R., "Simultaneous effects of coagulation and variable magnetic field on peristaltically induced motion of Jeffrey nanofluid containing gyrotactic microorganism", Microvascular Research. 2017, 110, 32-42.

[9] Bhatti, M.M., Zeeshan, A., Ellahi, R. and ljaz, N., "Heat and mass transfer of two-phase flow with Electric double layer effects induced due to peristaltic propulsion in the presence of transverse magnetic field", Journal of Molecular Liquids. 2017, 230, 237-246.

[10] Devakar, M. and lyengar, T.K.V., "Run up flow of an incompressible couple stress fluid between parallel plates", NonLinear Analysis: Modelling and Control. 2010, 15(1), 29-37.

[11] Devakar, M. and lyengar, T.K.V., "Stokes' problems for an incompressible couple stress fluid”, Non-linear Analysis: Modelling and Control. 2008, 1(2), 181-190.

[12] Devakar, M., Sreenivasu, D. and Shankar, B., "Analytical solutions of couple stress fluid flows with slip boundary conditions", Alexandria Engineering Journal. 2014, 53(3), 723-730.

[13] Devakar, M., Sreenivasu, D. and Shankar, B., "Analytical solutions of some fully developed flows of couple stress fluid between concentric cylinders with slip boundary conditions", International Journal of Engineering Mathematics. 2014, 785396.

[14] Ellahi, R., Bhatti, M.M., Fetecau, C. and Vafai, K., "Peristaltic flow of couple stress fluid in a non-uniform rectangular duct having compliant walls", Communications in Theoretical Physics. 2016, 65(1), 66.

[15] Ellahi, R., Bhatti, M.M. and Pop, I., "Effects of hall and ion slip on MHD peristaltic flow of Jeffrey fluid in a non-uniform rectangular duct", International Journal of Numerical Methods for Heat and Fluid Flow. 2016, 26(6), 1802-1820.

[16] Ellahi, R., Bhatti, M.M. and Khalique, C.M., "Threedimensional flow analysis of Carreau fluid model induced by peristaltic wave in the presence of magnetic field", Journal of Molecular Liquids. 2017, 241, 1059-1068.

[17] Ellahi, R., Raza, M. and Akbar, N.S., "Study of peristaltic flow of nanofluid with entropy generation in a porous medium", Journal of Porous Media. 2017, 20(5), 461-478.
[18] Hayat, T., Javed, M. and Hendi, A.A., "Peristaltic transport of viscous fluid in a curved channel with compliant walls" International Journal of Heat and Mass Transfer. 2011, 54, 1615-1621.

[19] Hayat, T., Saleem, A., Tanveer, A. and Alsaadi, F., “Numerical analysis for peristalsis of Williamson nanofluid in presence of an endoscope", International Journal of Heat and Mass Transfer. 2017, 114, 395-401.

[20] Hayat, T., Aslam, N., Alsaedi, A. and Rafiq, M., "Numerical analysis for endoscope and Soret and Dufour effects on peristalsis of Prandtl fluid", Results in Physics. 2017, 7, 28552864.

[21] Hina, S., Mustafa, M. and Hayat, T., "On the exact solution for peristaltic flow of couple-stress fluid with wall properties", Bulgarian Chemical Communications. 2015, 47(1), 30-37.

[22] Hina, S., Mustafa, M., Hayat, T. and Alsaedi, A., "Peristaltic flow of couple-stress fluid with heat and mass transfer: An application in biomedicine", Journal of Mechanics in Medicine and Biology. 2015, 15(4), 1550042.

[23] Hina, S., Mustafa, M., Hayat, T. and Alsaedi, A., "Peristaltic flow of Powell-Eyring fluid in curved channel with heat transfer: A useful application in biomedicine", Computer Methods and Programs in Biomedicine. 2016, 135, 89-100.

[24] Hina, S., Mustafa, M., Hayat, T. and Alsaedi, A., "Peristaltic transport of Powell-Eyring fluid in a curved channel with heat/mass transfer and wall properties", International Journal of Heat and Mass Transfer. 2016, 101, 156-165.

[25] Hina, S. and Nadeem, S., "Analysis of combined convective and viscous dissipation effects for peristaltic flow of Rabinowitsch fluid model”, Journal of Bionic Engineering. 2017, 14(1), 182-190.

[26] ljaz, S. and Nadeem, S., "A balloon model examination with impulsion of Cu-nanoparticles as drug agent through stenosed tapered elastic artery", Journal of Applied Fluid Mechanics. 2017, 10(6), 1773-1783.

[27] Khan, N.A., Mahmood, A. and Asmat A., "Approximate solution of couple stress fluid with expanding or contracting porous channel”, Engineering Computations. 2013, 30(3), 399-408.

[28] Khan, A., Muhammad, S., Ellahi, R. and Zia, Q., "Bionic study of variable viscosity on MHD peristaltic flow of pseudoplastic fluid in an asymmetric channel", Journal of Magnetics. 2016, 21(2), 273-280.

[29] Khan, A., Usman, H., Vafai, K. and Ellahi, R., "Study of peristaltic flow of magnetohydrodynamic Walter's B fluid with slip and heat transfer", Scientia Iranica. 2016, 23(6), 2650-2662.

[30] Mekheimer, Kh.S. and Abd elmaboud, Y., "Peristaltic flow of a couple stress fluid in an annulus: Application of an endoscope", Physica A: Statistical Mechanics and its Applications. 2008, 387(11), 2403-2415.

[31] Mekheimer, Kh.S. and Abd elmaboud, Y., "The influence of heat transfer and magnetic field on peristaltic transport of a Newtonian fluid in a vertical annulus: Application of an endoscope", Physics Letters A. 2008, 372(10), 1657-1665.

[32] Nadeem, S. and Akram, S., "Peristaltic flow of a Jeffrey fluid in a rectangular duct", Nonlinear Analysis: Real World Applications. 2010, 11(5), pp. 4238-4247.

[33] Nadeem, S. and Akbar, N.S., "Influence of temperature dependent viscosity on peristaltic transport of a Newtonian fluid: Application of an endoscope", Applied Mathematics and Computation. 2010, 216(12), 3606-3619. 
[34] Nadeem, S. and Akbar, N.S., "Influence of heat transfer and variable viscosity in vertical porous annulus with peristalsis" Journal of Porous Media. 2011, 14(10), 849-863.

[35] Nadeem, S., Akbar, N.S., Naheeda, B. and Sadaf, A., "Influence of heat and mass transfer on peristaltic flow of a third order fluid in a diverging tube", Communications in nonlinear Science and Numerical Simulation. 2010, 15(10), 2916-2931.

[36] Nadeem, S., Akbar, N.S., Hayat, T. and Hendi, A.A., "Peristaltic flow of Walter's B fluid in endoscope", Applied Mathematics and Mechanics. 2011, 32(6), 689-700.

[37] Nadeem, S. and Hina, S., "Trapping study of nanofluids in an annulus with cilia”, AIP Advances. 2015, 5, 127204.

[38] Nagarani, P. and Lewis, A., "Peristaltic flow of a Casson fluid in an annulus”, Korea-Australia Rheology Journal. 2012, 24(1), 1-9.

[39] Ramana Murthy, J.V. and Srinivas, J., "First and second law analysis for the MHD flow of two immiscible couple stress fluids between two parallel plates", Heat Transfer Asian Research. 2014, 43, 1-20.

[40] Ramesh, K. and Devakar, M., "Effects of heat and mass transfer on the peristaltic transport of MHD couple stress fluid through porous medium in a vertical asymmetric channel" Journal of Fluids. 2015, 2015, 163832.

[41] Ramesh, $K$ and Devakar, M., "The effects of endoscope and heat transfer on the peristaltic flow of a second grade fluid in an inclined tube", Journal of Mechanics in Medicine and Biology. 2016, 16, 1650057.

[42] Ramesh, K. and Devakar, M., "Magnetohydrodynamic peristaltic flow of Pseudoplastic fluid in a vertical asymmetric channel through porous medium with heat and mass transfer”, Iranian Journal of Science and Technology, Transactions A: Science. 2017, 41(1), 257-272.

[43] Ramzan, M., Farooq, M., Alsaedi, A. and Hayat, T., "MHD threedimensional flow of couple stress fluid with Newtonian heating”, The European Physical Journal Plus. 2013, 128, 49.

[44] Shahzadi, I. and Nadeem, S., "Role of inclined magnetic field and copper nanoparticles on peristaltic flow of nanofluid through inclined annulus: Application of the clot model", Communications in Theoretical Physics. 2017, 67(6), 704.
[45] Shahzadi, I. and Nadeem, S., "Inclined magnetic field analysis for metallic nanoparticles submerged in blood with convective boundary condition", Journal of Molecular Liquids. 2017, 230, 61-73.

[46] Shahzadi, I., Sadaf, H., Nadeem, S. and Saleem, A., “Biomathematical analysis for the peristaltic flow of single wall carbon nanotubes under the impact of variable viscosity and wall properties", Computer Methods and Programs in Biomedicine. 2017, 139, 137-147.

[47] Shahzadi, I., Nadeem, S. and Rabiei, F., "Simultaneous effects of single wall carbon nanotube and effective variable viscosity for peristaltic flow through annulus having permeable walls", Results in Physics. 2017, 7, 667-676.

[48] Srinivasacharya, D. and Kaladhar, K., "Mixed convection flow of chemically reacting Couple stress fluid in a vertical channel with Soret and Dufour effects", International Journal for Computational Methods in Engineering Science and Mechanics. 2014, 15, 413-421.

[49] Srinivasacharya, D and Srikanth, D., "Steady streaming effect on the flow of a couple stress fluid through a constricted annulus", Archives of Mechanics. 2012, 64(2), 137-152.

[50] Srinivasacharya, D. Srinivasacharyulu, N. and Odelu, O., "Flow and heat transfer of couple stress fluid in a porous channel with expanding and contracting walls", International Communications in heat and mass Transfer. 2009, 36, 180-185.

[51] Srivastava, L.M., "Flow of couple stress fluid through stenotic blood vessels", Journal of Biomechanics. 1985, 18(7), 479485.

[52] Stokes, V.K., "Couple stresses in fluids”, Physics of Fluids. 1966, 9(9), 1709-1715.

[53] Tripathi, D., "Peristaltic transport of fractional Maxwell fluids in uniform tubes: Applications in endoscopy”, Computers \& Mathematics with Applications. 2011, 62(3), 1116-1126.

[54] Vajravelu, K., Radhakrishnamacharya, G. and Radhakrishnamurty, V., "Peristaltic flow and heat transfer in a vertical porous annulus, with long wave approximation”, International Journal of Non-Linear Mechanics. 2007, 42(5), 754-759.

[55] Wang, Y. Ali, N. Hayat, T. and Oberlack, M., "Peristaltic motion of a magnetohydrodynamic micropolar fluid in a tube", Applied Mathematical Modelling. 2011, 35(8), 3737-3750. 